\title{
East-West Effect on VLF Mode Transmission Across the Earth's Magnetic Field ${ }^{1}$
}

\author{
D. Dobrott and A. Ishimaru ${ }^{2}$
}

(July 5, 1960)

\begin{abstract}
The effect of a constant transverse magnetic field is analyzed for the propagation of VLF electromagnetic waves about the earth. These waves are considered to be propagating by modes in a parallel plate waveguide. The lower boundary of the guide is considered to be a perfect conductor, while the upper boundary is assumed to be a sharply defined, semi-infinite, homogeneous plasma with a constant magnetic field applied. The source of these waves is an infinite number of short, horizontal, cophased dipoles, uniformly spaced parallel to the constant magnetic field vector. Admittance relations are derived for the upper boundary of the guide by considering the waves to be of grazing incidence. It is found that these admittance parameters depend on the direction of propagation. These admittance parameters are plotted versus frequency for various heights. Expressions for eigenvalues and the eigenfunctions are found as functions of the admittance. By employing a two dimensional Green's function, the amplitudes of the various modes due to the dipole source are evaluated. It is noted that the propagation constants differ depending on the direction of propagation, thus offering an explanation of the east-west effect of VLF transmission. A numerical example is calculated and field strength versus distance values are found to correspond to some experimental results.
\end{abstract}

\section{Introduction}

The mode theory of VLF radio transmission has evolved from the study of electromagnetic wave propagation between the earth and the ionosphere. Wait [1] ${ }^{3}$ and others $[2,3]$ have solved the VLF electromagnetic problem using mode theory and assuming a sharply bounded ionosphere, while neglecting the earth's magnetic field. Friedman $[4,5]$ has made a modal analysis which differ in method from that of Wait, but arrives at similar conclusions. Friedman's analysis utilized an upper boundary of scalar impedance $Z$.

All of these solutions have the same propagation characteristics regardless of the direction of propagation. However, Crombie [6] has noted evidence that VLF propagation differs depending on whether the propagation is from east to west, or from west to east. He also indicated that this so-called "eastwest effect" may be partly due to the earth's magnetic field. Recently, Wait $[7,8]$ and Crombie $[6,9]$ have shown analytically that the rate of attenuation for east-west propagation behaves as had been indicated by the latter author.

This paper attempts to show analytically the eastwest effect on the basis of mode theory and ionosphere-freespace boundary admittance. Because of the presence of the earth's magnetic field, the admittance of the sharply bounded ionosphere is not a scalar, but a tensor. Further, because of these magnetic effects, the tensor depends upon the direction of propagation.

\footnotetext{
1 Contribution from Boeing Scientific Research Laboratory, Seattle, Wash. 2 Present address of Professor A. Ishimaru: Department of Electrical Engineering, University of Washington, Seattle, Wash.
}

3 Figures in brackets indicate the literature references at the end of this paper.
The solution of the radiation from a dipole in this parallel plate waveguide with anisotropic boundary conditions is, in general, difficult. However, one may solve an analogous two-dimensional problem. This simplifies the arithmetic while maintaining the qualitative structure of the three-dimensional case.

The two-dimensional problem corresponds to the source of electromagnetic waves being an infinite number of horizontal, cophased, short dipoles, uniformly spaced parallel to the direction to the earth's magnetic field. This situation is displayed in figure 1.

In the sections that follow, the radiation from these sources are examined in detail. A numerical example is shown also.

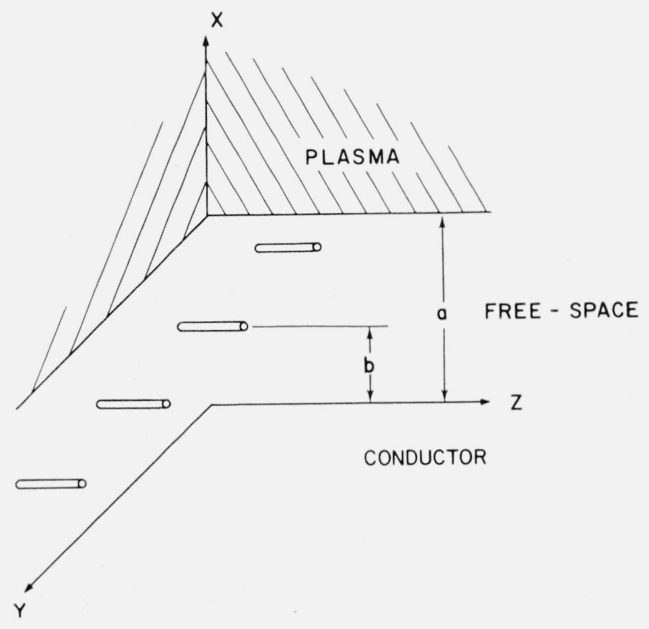

Figure 1. Cophased sources for TM waves. 


\section{Dielectric Tensor of Magnetically In- fluenced Plasma}

The permittivity of an ionized gas may be determined by examining the ion motion under the influence of electromagnetic waves. Certain restrictions must be placed on this gas if the evaluation of the equation of motion is to remain simple.

For the purpose of this analysis, the gas will be considered to be a homogeneous plasma. That is, the gas is uniform throughout and the number of positive charges is equal to the number of negative charges. The plasma exists in a state where collisions are relatively few. Since the positive and neutral particles have more mass than the electrons, their mobilities are correspondingly smaller and it shall be considered that their velocity is zero. Further, the number of electrons per cubic meter $N$, does not vary appreciably with time. Thus the behavior of a volume element of gas is the same as that of an "average" electron obeying the laws of classical dynamics. These are the assumptions of the "Magneto-Ionic Theory" [10].

In the special case considered in figure 1, where a magnetic field is considered perpendicular to the direction of propagation, the ionosphere's relative dielectric tensor is given by, [11]

$$
\begin{aligned}
& \overline{\bar{\epsilon}}=\left[\begin{array}{ccr}
\epsilon_{2} & 0 & -j \eta \\
0 & \epsilon_{1} & 0 \\
j \eta & 0 & \epsilon_{2}
\end{array}\right], \\
& \epsilon_{1}=1+\frac{\omega_{p}^{2}}{j \omega \nu-\omega^{2}}, \\
& \epsilon_{2}=1+\frac{\omega_{p}^{2}(j \omega+\nu)}{j \omega\left[\omega_{c}^{2}+(j \omega+\nu)^{2}\right]}, \\
& \eta=-\frac{\omega_{c} \omega_{p}^{2}}{\omega\left[\omega_{c}^{2}+(j \omega+\nu)^{2}\right]} \cdot
\end{aligned}
$$

In expressions 2.2, 2.3 and $2.4 \omega_{p}$ is the plasma frequency, $\nu$ is the collision frequency, $\omega_{c}$ is the cyclotron frequency, and $\omega$ is the frequency of the electromagnetic wave propagating in the plasma region.

Using 2.1, one is able to solve for the electromagnetic fields in the plasma region. The solution of the fields is required to examine the conditions at the plasma-freespace interface.

\section{Admittance Tensor of Plasma at Interface}

In order to solve for the electromagnetic fields for the problem described by section 1 , one must first consider the restrictions placed upon the problem by the particular geometry. Since the sources are considered to be uniformly distributed along the $y$ direction axis, there is no $y$-variation for the fields.
Further, the nature of these sources produces only transverse-magnetic waves. Considering these facts and eq 2.1, Maxwell's equations in the plasma become,

$$
\begin{aligned}
& \frac{\partial E_{z}}{\partial x}-\frac{\partial E_{x}}{\partial z}=j \omega \mu_{0} H_{y}, \\
& \frac{\partial H_{y}}{\partial z}=-j \omega \epsilon_{0}\left(\epsilon_{2} E_{x}-j \eta E_{z}\right), \\
& \frac{\partial H_{y}}{\partial x}=j \omega \epsilon_{0}\left(j \eta E_{x}+\epsilon_{2} E_{z}\right) .
\end{aligned}
$$

Solving eqs 3.1 in terms of the longitudinal electric field to obtain the following equation

$$
\left[\frac{\partial^{2}}{\partial x^{2}}+\frac{\partial^{2}}{\partial z^{2}}+k^{2} \frac{\left(\epsilon_{2}^{2}-\eta^{2}\right)}{\epsilon_{2}}\right] E_{z}=0 .
$$

The solution to this equation is of the form

$$
E_{z}=K_{1} \epsilon^{-p x} \epsilon^{ \pm \Upsilon^{z}}
$$

where $p$ and $\gamma$ are, in general, complex and the plus or the minus sign designates a wave traveling in the negative $z$-direction or positive $z$-direction respectively.

Substituting eq 3.3. into 3.2 one obtains the characteristic equation of 3.2

$$
p^{2}+\gamma^{2}+k^{2} \frac{\left(\epsilon_{2}^{2}-\eta^{2}\right)}{\epsilon_{2}}=0 .
$$

In the free-space region, Maxwell's equations may be solved for the transverse-magnetic case in terms of the longitudinal electric field yielding the following homogeneous wave equation

$$
\left[\frac{\partial^{2}}{\partial x^{2}}+\frac{\partial^{2}}{\partial z^{2}}+k^{2}\right] E_{z}=0 \text {. }
$$

The solution for the homogeneous wave equation depends upon the boundary conditions, and since the tangential electric field must be zero at a perfect conductor, the solution is of the form,

$$
E_{z}=K_{2} \sin q x \epsilon^{ \pm \Upsilon z}
$$

where $q$ and $\gamma$ are complex.

Equation 3.6 has a corresponding characteristic equation given by,

$$
-q^{2}+\gamma^{2}+k^{2}=0 .
$$


From eqs 3.1 and 3.3 the fields in the plasma region are related by the following relations for the $+z$-direction,

$$
\begin{aligned}
& E_{x}=\frac{1}{j \omega \epsilon_{0}} \frac{\epsilon_{2} \gamma-j p \eta}{\epsilon_{2}^{2}-\eta^{2}} H_{y} \\
& E_{z}=\frac{1}{j \omega \epsilon_{0}} \frac{-j \eta \gamma-\epsilon_{2} p}{\epsilon_{2}^{2}-\eta^{2}} H_{y}
\end{aligned}
$$

for the -z-direction

$$
\begin{aligned}
& E_{x}=\frac{1}{j \omega \epsilon_{0}} \frac{-\epsilon_{2} \gamma-j p \eta}{\epsilon_{2}^{2}-\eta^{2}} H_{y} \\
& E_{z}=\frac{1}{j \omega \epsilon_{0}} \frac{j \eta \gamma-\epsilon_{2} p}{\epsilon_{2}^{2}-\eta^{2}} H_{p} .
\end{aligned}
$$

From the two tangential fields $E_{z}$ and $H_{y}$, an admittance relationship may be defined as

$$
Y_{0} Y_{12}=-\left.\frac{H_{y}}{E_{z}}\right|_{x=a} .
$$

From the two expressions of eqs 3.8 and relation 3.9, one may obtain two expressions for the admittance of the plasma boundary.

$$
Y_{\substack{e-w \\ w-e}}=\frac{j k\left(\epsilon_{2}^{2}-\eta^{2}\right)}{\epsilon_{2} p \pm j \eta \gamma}
$$

where $Y_{e-w}$ and $Y_{w-e}$ are the relative admittance parameters for east-west and west-east propagation, respectively.

If grazing incidence is considered, upon the plasmafreespace interface, it is found that $\gamma \rightarrow j k$, where the sign has already been determined by the form of eq 3.6. This corresponds to the far-field situation. If this condition were not imposed, the admittance would be a function of the free-space eigenvalue $q$. Thus one obtains the following two expressions for the admittance of the plasma boundary,

$$
Y_{\substack{e-w \\ w-e}}=\frac{j k\left(\epsilon_{2}^{2}-\eta^{2}\right)}{\epsilon_{2} p \mp \eta k} .
$$

The following restriction must be placed upon $p$,

$$
\operatorname{Re}(p)>0, \operatorname{Im}(p)>0 .
$$

With these restrictions in mind, the final expressions for the admittances in terms of the constituents of equation 2.1 are

$$
Y_{\substack{e-w \\ w-e}}=\frac{j\left(\epsilon_{2}^{2}-\eta^{2}\right)}{\epsilon_{2}\left[1-\frac{\epsilon_{2}^{2}-\eta^{2}}{\epsilon_{2}}\right]^{1 / 2} \mp \eta} .
$$

A numerical solution of these admittance parameters for height versus frequency is shown in figures 2 and 3. The values of collision frequency and electron density have been obtained from papers by M. Nicolet [12] and R. E. Houston [13], respectively.

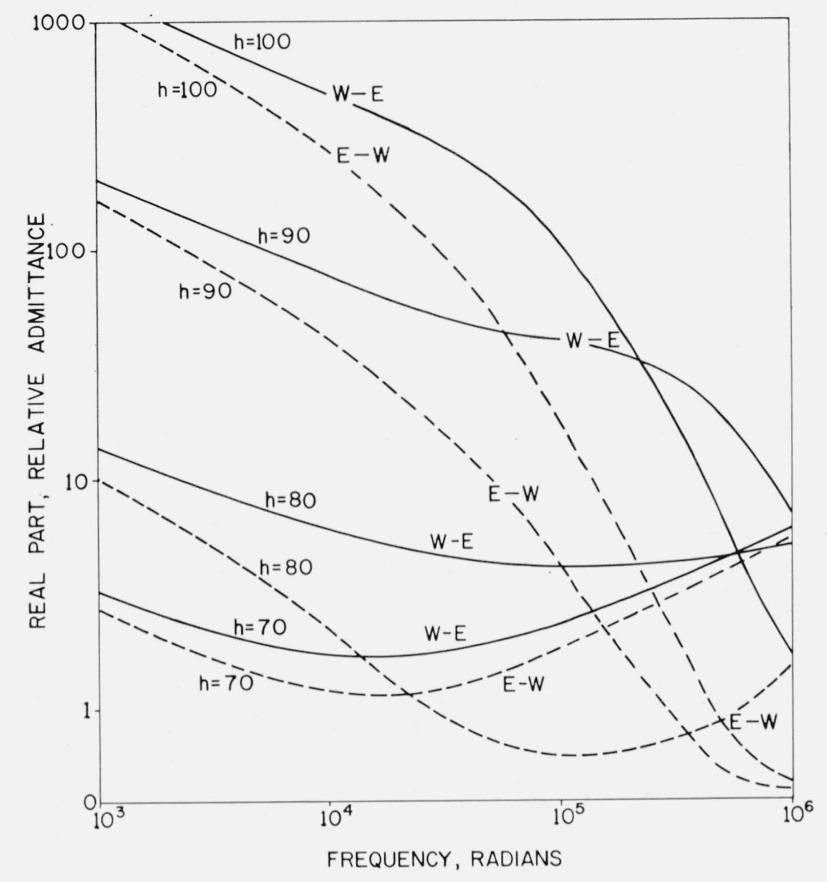

Figure 2. Real part of admittance $\left(\mathrm{Y}_{12}\right)$.

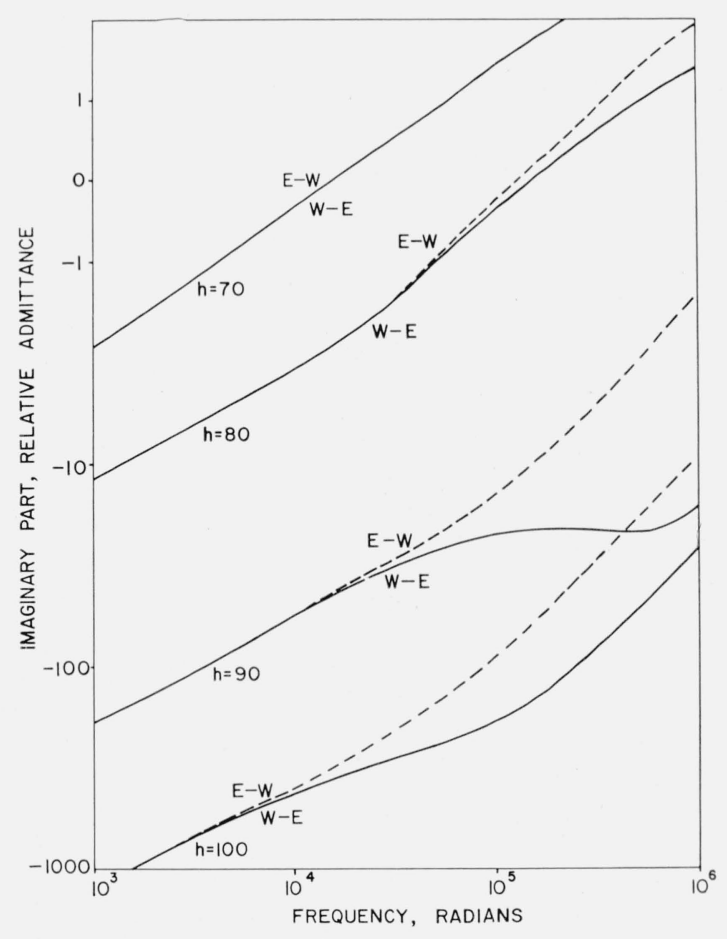

FIgURE 3. Imaginary part of admittance $\left(\mathrm{Y}_{12}\right)$. 


\section{Eigenvalues and Propagation Constant}

In order to solve for a general expression for the eigenvalues in terms of the admittance matrix for all waves, one must also solve Maxwell's equations for the transverse-electric waves. This solution, in terms of the longitudinal magnetic field $H_{z}$, is given as

$$
H_{z}=Y_{0} K_{3} \epsilon^{ \pm \gamma z} \cos q x .
$$

Recalling eq 3.6 and Maxwell's equations for TM and TE one can obtain,

$$
E_{y}=-\frac{j \omega \mu_{0}}{q} Y_{0} K_{3} \epsilon^{ \pm \gamma z} \sin q x
$$

and

$$
H_{y}=-\frac{j \omega \epsilon_{0}}{q} K_{2} \epsilon^{ \pm \gamma z} \cos q x .
$$

Relating these four fields at $x=a$ by the admittance matrix $\overline{\bar{Y}}$,

$$
\left[\begin{array}{l}
H_{y} \\
H_{z}
\end{array}\right]=Y_{0}\left[\begin{array}{ll}
Y_{11} & Y_{12} \\
Y_{21} & Y_{22}
\end{array}\right]\left[\begin{array}{l}
E_{y} \\
E_{z}
\end{array}\right] .
$$

Substituting the solutions $3.6,4.1,4.2$, and 4.3 into 4.4 and combining terms

$$
\left[\begin{array}{l}
\left(Y_{12} \sin q a-\frac{j k}{q} \cos q a\right)\left(\frac{j k}{q} Y_{11} \sin q a\right) \\
\left(Y_{22} \sin q a\right)\left(\cos q a+\frac{j k}{q} \sin q a\right)
\end{array}\right]\left[\begin{array}{l}
K_{2} \\
K_{3}
\end{array}\right]=0 .
$$

For 4.5 to be true, the determinant

$$
\left|\begin{array}{l}
\left(Y_{12} \sin q a-\frac{j k}{q} \cos q a\right)\left(\frac{j k}{q} Y_{11} \sin q a\right) \\
\left(Y_{22} \sin q a\right)\left(\cos q a+\frac{j k}{q} Y_{21} \sin q a\right)
\end{array}\right|=0 .
$$

Evaluating the determinant 4.6, the following general expression for the eigenvalues is obtained in terms of admittance parameters,

$$
\Delta_{y} \tan q a+\cot q a=-j \frac{q^{2} Y_{12}+k^{2} Y_{21}}{q k}
$$

where $\Delta_{y}=Y_{11} Y_{22}-Y_{12} Y_{21}$.

For the special parallel plate case under consideration, the only admittance parameter needed is $Y_{12}$. Thus eq 4.7 becomes

$$
\cot q a=-j \frac{q}{k} Y_{12}
$$

Rewriting relationship $4.8, g=g_{n}, n=1,2,3 \ldots$

$$
j z q_{n} a=\ln \left(1-\frac{2}{1+\frac{q n Y_{12}}{k}}\right)+j z n \pi .
$$

If the term $q_{n} / k \quad Y_{12}$ is large as is the case for the "electric" wall, the following approximation for the eigenvalue $q_{n}$ may be obtained.

$$
q_{n}=\frac{n \pi}{a}+\frac{j k a}{q_{n} a^{2} Y_{12}} .
$$

For the "zeroth mode" case, that is $n=0$,

$$
q_{0}=\left(\frac{j k}{a Y_{12}}\right)^{1 / 2},
$$

and if $n$ is greater than zero, $q_{n}$ may be approximated $n \pi$ in the right-hand side of 4.10 yielding,

$$
q_{n}=\frac{n \pi}{a}+\frac{j k}{n \pi Y_{12}} .
$$

The propagation constant

$$
\gamma_{n}=\left(q_{n}^{2}-k^{2}\right)^{1 / 2}
$$

may be obtained from the results of 4.11 and 4.12 . Restrictions on 4.13 are that

$$
\operatorname{Re}\left(\gamma_{n}\right)>0, \operatorname{Im}\left(\gamma_{n}\right)>0
$$

when 4.13 is evaluated for $n=0$, the attenuation constant, $\operatorname{Re}\left(\gamma_{n}\right)=\alpha_{n}$ is very small, and for $n>2$, the phase constant, $\operatorname{Im}\left(\gamma_{n}\right)=\beta_{n}$, is small representing evanescent modes.

One can see that if $Y_{12} \rightarrow \infty$, as in the case of a perfect conductor, expression 4.12 reduce to the familiar result, $n \pi / a$. Expressions 4.11 and 4.12 are used, along with the data of Houston and Nicolet, to plot mode decibel loss versus distance on figure 4 for $n=0,1,2$. The numerical values for the admittance used were

$$
\begin{aligned}
& Y_{E-W}=22.5-37.5 j, \\
& Y_{W-E}=56.5-41.5 j .
\end{aligned}
$$

This corresponds to a radian frequency of $\omega=2 \times 10^{4}$ and a height of $90 \mathrm{~km}$.

A synopsis of experimental results by Crombie [6] indicates that the ratio of electric field strengths

$$
\frac{W-E}{E-W}>1
$$

which is certainly true for the numerical case calculated and graphed on figure 4 . 


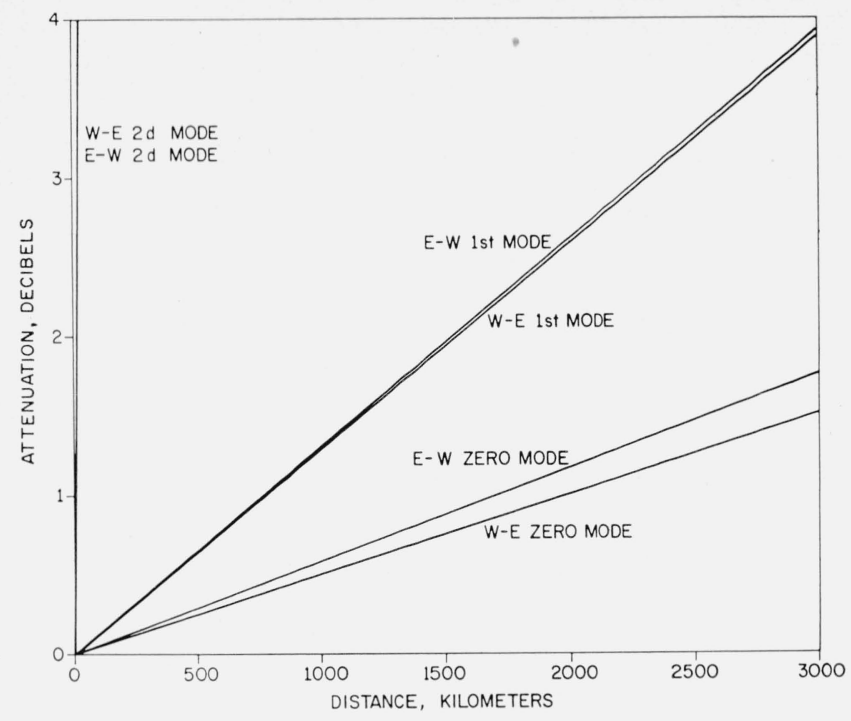

Figurfi 4. Attenuation versus distance.

\section{Mode Solution-Source Present}

With the source of current moment $I L$ eq 3.5 becomes,

$$
\left(\frac{\partial^{2}}{\partial x^{2}}+\frac{\partial^{2}}{\partial z^{2}}+k^{2}\right) E_{z}=j \omega \mu_{0} I L \delta(x-b) \delta(z)
$$

where $\delta(x-b)$ and $\delta(z)$ are Dirac "delta-functions." This problem is analogous to the Green's function problem if one relates the longitudinal electric field to the Green's function as $E_{z}=-j \omega \mu_{0} I L G$, the corresponding Green's function problem may be solved,

$$
\left(\frac{\partial^{2}}{\partial x^{2}}+\frac{\partial^{2}}{\partial z^{2}}+k^{2}\right) G=-\delta(x-b) \delta(z) .
$$

The boundary conditions for the Green's function are the same as for the electric field $E_{z}$

$$
\begin{aligned}
\left.G\right|_{x=0} & =0 \\
\left.\left(G^{\prime}+Y G\right)\right|_{x=a} & =0 .
\end{aligned}
$$

It has been shown in section 4 that the solution to the homogeneous wave equation for the longitudinal electric field and hence the Green's function is of the form

$$
G=\sum_{0}^{\infty} n A_{n} \epsilon^{-\gamma_{n}|z|} \sin q_{n} x .
$$

Thus if 5.2 is true we must be able to expand the delta-function in terms of eigenvectors, $[14,15]$

$$
\delta(x-b)=\sum_{0}^{\infty} n B_{n} \sin q_{n} x .
$$

Multiplying through by $\sin q_{m} x$ and integrating over the range of $x$, an expression for $B_{n}$

$$
B_{n}=\frac{4 q_{n} \sin q_{n} b}{\left(2 q_{n} a-\sin 2 q_{n} a\right)} .
$$

Making the substitution

$$
r_{n}=\frac{4 q_{n}}{\left(2 q_{n} a-\sin 2 q_{n} a\right)}
$$

where $r_{n}$ is often called the normalization factor. Integrating 5.2 over the source with respect to $z$ yields

and

$$
A_{n}=\frac{r_{n} \sin q_{n} b}{2 \gamma_{n}}
$$

$$
G=\sum_{0}^{\infty} n \frac{2 q_{n}}{\gamma_{n}\left(2 q_{n} a-\sin 2 q_{n} a\right)} \epsilon^{-\gamma_{n}|z|} \sin q_{n} b \sin q_{n} x .
$$

Therefore the expression for the electric field is given by

$$
E_{z}=\sum_{0}^{\infty} n \frac{j \omega \mu_{0} 2 I L q_{n}}{\gamma_{n}\left(2 q_{n} a-\sin 2 q_{n} a\right)} \epsilon^{-\gamma_{n}|z|} \sin q_{n} b \sin q_{n} x .
$$

The other fields may be evaluated for Maxwell's equations for the TM case.

The expression for the vertical component of the electric field with a "normalized" current is given as

$$
E_{x}=\sum_{0}^{\infty} n \frac{\sin q_{n} b}{2 q_{n} a-\sin 2 q_{n} a} \epsilon^{-\gamma_{n}|z|} \cos q_{n} x .
$$

If the transmitter is in an airplane at a height of $a / 4$ and the receiver is on the ground, 5.11 becomes,

$$
E_{x}=\sum_{0}^{\infty} n \frac{\sin q_{n}\left(\frac{a}{4}\right)}{2 q_{n} a-\sin 2 q_{n} a} \epsilon^{-\gamma_{n}|z|}
$$

For the first three modes 5.12 is of the form

$$
E_{x}=C_{0} \epsilon^{-\gamma_{0} z}+C_{1} \epsilon^{-\gamma_{1} z}+C_{2} \epsilon^{-\gamma_{2} z}
$$

where

$$
C_{n}=\frac{\sin q_{n}\left(\frac{a}{4}\right)}{2 q_{n} a-\sin 2 q_{n} a} .
$$

The magnitude of the field strength at the receiver is given by the expression,

$$
\begin{aligned}
\left|E_{x}\right| & =\left\{\left|C_{0}\right|^{2} \epsilon^{-\alpha_{0} z}+\left|C_{1}\right|^{2} \epsilon^{-2 \alpha_{1} z}+\left|C_{2}\right|^{2} \boldsymbol{\epsilon}^{-2 \alpha_{2} z}\right. \\
& +2\left|C_{0}\right|\left|C_{1}\right| \epsilon^{-\left(\alpha_{0}+\alpha_{1}\right) z} \cos \left[\left(\beta_{0}-\beta_{1}\right) z+\left(\bar{C}_{0}-\bar{C}_{1}\right)\right] \\
& +2\left|C_{0}\right|\left|C_{2}\right| \epsilon^{-\left(\alpha_{0}+\alpha_{2}\right) z} \cos \left[\left(\beta_{0}-\beta_{2}\right) z+\left(\bar{C}_{0}-\bar{C}_{2}\right)\right] \\
& \left.\left.+2\left|C_{1}\right|\left|C_{2}\right| \epsilon^{-\left(\alpha_{1}+\alpha_{2}\right) z} \cos \left[\left(\beta_{1}-\beta_{2}\right) z+\left(\bar{C}_{1}-\bar{C}_{2}\right)\right]\right\}\right\}^{1 / 2}
\end{aligned}
$$


where $\left|C_{n}\right|$ and $\bar{C}_{n}$ are the magnitude and argument of $C_{n}$, respectively.

Equation 5.15 is plotted on figure 5. These results depend not only on attenuation, but also on field strength magnitude which in turn depends on the direction of propagation and the height of the transmitting antenna. This corresponds qualitatively with the far field experimental results mentioned by Crombie [6].

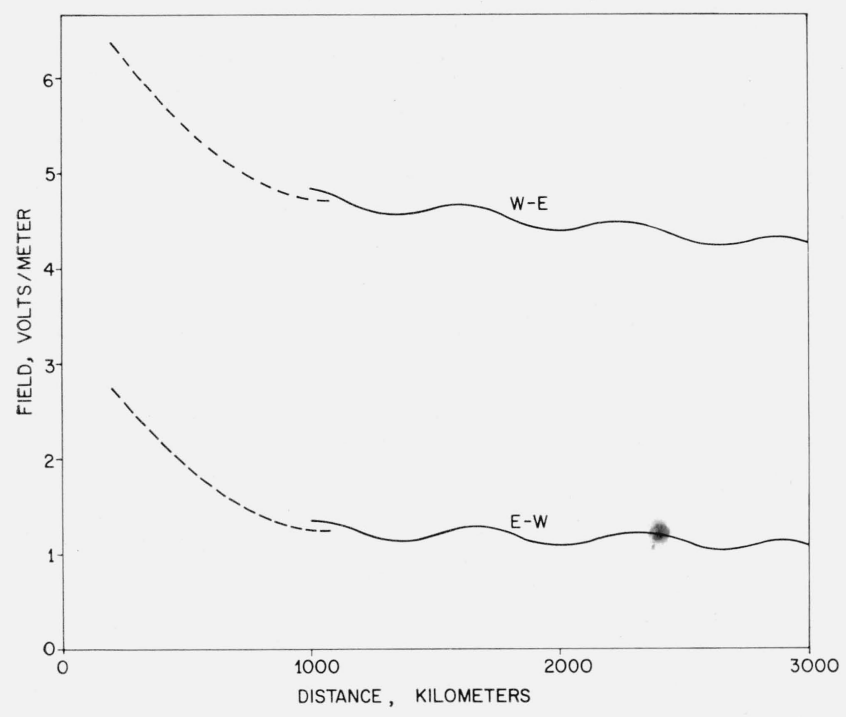

Figure 5. Field strength versus distance.

\section{Conclusion}

The effect on east-west, west-east propagation of electromagnetic transverse magnetic waves across the earth's magnetic field has been analyzed as a boundary value problem. Certain idealizations were made to solve this problem. Admittance relationships were derived from field relations existing in a homogeneous anisotropic plasma, and it was found that the admittance parameters depended upon the direction of propagation. These parameters were plotted versus frequency for various heights. Expressions for the eigenvalues were derived. The attenuation over distance was plotted for a special case. It was found that the ratio of the electric field for the individual modes of west-east/east-west propagation was greater than one. This corresponds to some experimental data. A two dimensional Green's function was derived and a numerical calculation of the corresponding electric field strength was plotted versus distance.

The authors express appreciation to the Scientific Research Laboratory and the computer facilities group of the Boeing Airplane Company. Special thanks go to Dr. Bernard Friedman of the University of California for the valuable conversations with the authors while this paper was being written, and to Dr. James R. Wait for his critical and stimulating reviews of this work.

\section{References}

[1] James R. Wait, The mode theory of VLF ionospheric propagation for finite ground conductivity, Proc. IRE 45, 760 (1957).

[2] Kenneth Budden, The waveguide mode theory of the propagation of very-low-frequency radio waves, Proc. IRE 45, 772 (1957).

[3] Irving W. Yabroff, Reflection at a sharply-bounded ionosphere, Proc. IRE 45, 750 (1957).

[4] Bernard Friedman, Low frequency propagation, Boeing Airplane Co. Document D2-2302 (Nov. 1957).

[5] Bernard Friedman, Low frequency propagation in the ionosphere, Boeing Airplane Co. Document D1-820003 (June 1959).

[6] D. D. Crombie, Differences between the east-west and west-east propagation of VLF signals over long distances, J. Atmospheric and Terrest. Phys. 12, 110 (1958).

[7] James R. Wait, Terrestrial propagation of VLF radio waves - a theoretical investigation, J. Research NBS 64D, 153 (1960)

[8] James R. Wait and K. Spies, Influence of earth's curvature and terrestrial magnetic field on VLF propagation, J. Geophys. Research (Aug. 1960).

[9] D. D. Crombie, On the mode theory of very-low-frequency propagation in the presence of a transverse magnetic field, J. Research NBS 64D, 265 (1960).

[10] J. A. Ratcliffe, The magneto-ionic theory and its applications to the ionosphere (Cambridge Univ. Press, Cambridge, England, 1959).

[11] D. R. Dobrott, Effects in VLF mode transmission due to earth's magnetic field, Master's Thesis, University of Washington, Seattle, Wash., (1960).

[12] M. Nicolet, Aeronomic conditions in the mesosphere and lower thermosphere, Penn. State Univ. Ionospheric Research Rept. No. 102 (Apr. 1958).

[13] R. E. Houston, The effect of certain solar radiations in the lower ionosphere, Penn. State Univ. Ionospheric Research Rept. No. 95 (July 1957).

[14] Bernard Friedman, Principles and techniques of applied mathematics (John Wiley and Sons, New York, N.Y., 1957).

[15] Arnold Sommerfeld, Partial differential equations in physics, Lectures on theoretical physics, Vol. VI (Academic Press, Inc., New York, N.Y., 1949).

(Paper 65D1-102) 